This copy is for your personal, non-commercial use only.

If you wish to distribute this article to others, you can order high-quality copies for your colleagues, clients, or customers by clicking here.

Permission to republish or repurpose articles or portions of articles can be obtained by following the guidelines here.

The following resources related to this article are available online at www.sciencemag.org (this information is current as of June 11, 2012 ):

Updated information and services, including high-resolution figures, can be found in the online version of this article at:

http://www.sciencemag.org/content/313/5783/84.full.html

Supporting Online Material can be found at:

http://www.sciencemag.org/content/suppl/2006/07/03/313.5783.84.DC1.html
A list of selected additional articles on the Science Web sites related to this article can be found at:
http://www.sciencemag.org/content/313/5783/84.full.html\#related
This article has been cited by 82 article(s) on the ISI Web of Science
This article has been cited by 1 articles hosted by HighWire Press; see:
http://www.sciencemag.org/content/313/5783/84.full.html\#related-urls
This article appears in the following subject collections:
Chemistry
http://www.sciencemag.org/cgi/collection/chemistry 


\section{Photoproduction of Proton Gradients with $\pi$-Stacked Fluorophore Scaffolds in Lipid Bilayers}

\author{
Sheshanath Bhosale, ${ }^{1}$ Adam L. Sisson, ${ }^{1}$ Pinaki Talukdar, ${ }^{1}$ Alexandre Fürstenberg, ${ }^{2}$ \\ Natalie Banerji, ${ }^{2}$ Eric Vauthey, ${ }^{2}$ Guillaume Bollot, ${ }^{1}$ ]iri Mareda, ${ }^{1}$ Cornelia Röger, ${ }^{3}$ \\ Frank Würthner, ${ }^{3}$ Naomi Sakai, ${ }^{1}$ Stefan Matile ${ }^{1 *}$
}

Rigid $p$-octiphenyl rods were used to create helical tetrameric $\pi$-stacks of blue, red-fluorescent naphthalene diimides that can span lipid bilayer membranes. In lipid vesicles containing quinone as electron acceptors and surrounded by ethylenediaminetetraacetic acid as hole acceptors, transmembrane proton gradients arose through quinone reduction upon excitation with visible light. Quantitative ultrafast and relatively long-lived charge separation was confirmed as the origin of photosynthetic activity by femtosecond fluorescence and transient absorption spectroscopy. Supramolecular self-organization was essential in that photoactivity was lost upon rod shortening (from $p$-octiphenyl to biphenyl) and chromophore expansion (from naphthalene diimide to perylene diimide). Ligand intercalation transformed the photoactive scaffolds into ion channels.

I $\mathrm{n}$ biological systems, light harvesting uses the energy in photons to drive chemical reactions. Although many biomimetic and supramolecular compounds have been devised to collect photons, the output of these systems often consists of photons of lower energy. The outstanding challenge is to devise schemes in which acceptor chromophores can transfer the charge carrier (electron or hole) to generate a chemical product (for example, $\mathrm{H}^{+}$or $\mathrm{O}_{2}$ ) before relaxation to the initial state occurs.

In proteins, a series of chromophores is used to separate charges and avoid back reactions (1-3). In smaller biomimetic assemblies, an alternative strategy is to transfer charge to electron donors and acceptors in solution, provided that these donors and acceptors can be spatially separated and that these transfers occur faster than the charge recombination (4-7). We report the design, synthesis, and evaluation of blue, red-fluorescent rigid-rod photosystems (1), in which the $\pi$-stack apparently supports sufficient charge-separation lifetimes for conversion into chemical gradients (Fig. 1).

The construction of complex and multifunctional $\pi$-architecture is difficult because of the poor directionality of $\pi, \pi$-interactions $(8-18)$. Apart from a central role in oligonucleotide chemistry and biology (18), cofacial $\pi$-stack architecture contributes very little to electron transfer in biological (1-3) and synthetic (4-7) photosystems, not to speak of the structure of biological or synthetic ion channels or pores (19). Recently, we have demonstrated that the rational design of refined transmembrane $\pi$-stack archi-

${ }^{1}$ Department of Organic Chemistry, ${ }^{2}$ Department of Phys ical Chemistry, University of Geneva, Geneva, Switzerland. ${ }^{3}$ Institut für Organische Chemie, Universität Würzburg, Am Hubland, D-97074 Würzburg, Germany.

${ }^{*}$ To whom correspondence should be addressed. E-mail: stefan.matile@chiorg.unige.ch tecture is feasible with the use of rigid-rod molecules as preorganizing scaffolds $(20,21)$. This strategy was applied not only to create photosystem 1 but also to introduce multifunctionality; that is, to enable photosystem $\mathbf{1}$ to open up into ion channel $\mathbf{2}$ in response to the intercalation of ligands $\mathbf{3}$ (Fig. 1).

Photosystem 1 was prepared by self-assembly of four p-octiphenyls (4), each bearing eight

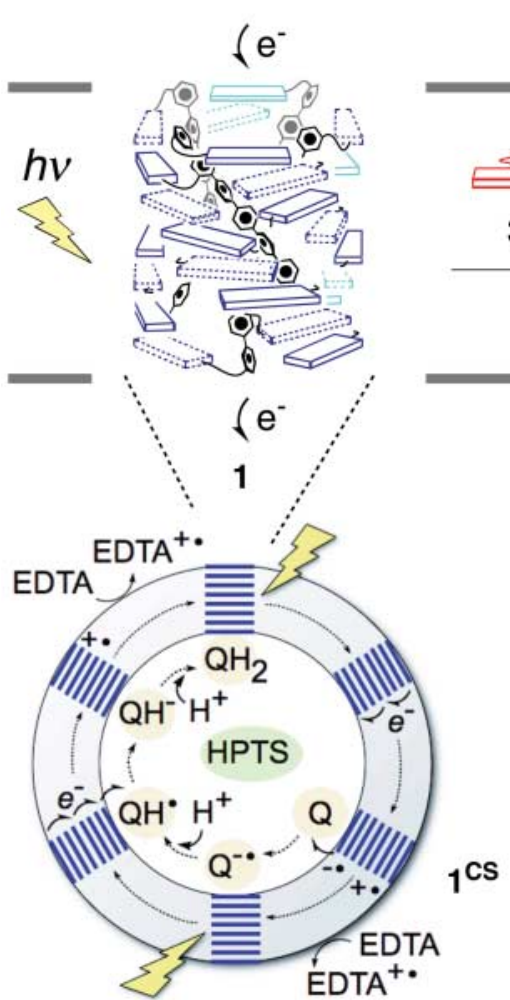

core-substituted naphthalene diimides (NDIs) (22) along the rigid-rod scaffold (Fig. 2 and scheme S1). The NDI octamer 4 was synthesized from simple commercial starting materials $\mathbf{5}$ to $\mathbf{1 0}$ in 20 steps altogether. Pyrene $\mathbf{5}$ was oxidized to the core-chlorinated dianhydride to allow for the introduction of terminal (6 and 7) and central (8) amines before attachment along the $p$-octiphenyl rod made from four biphenyls (10) (23). The following control molecules were synthesized in a similar manner: NDI dimer 11, NDI monomer 12, perylene diimide (PDI) octamer 13, and PDI dimer 14 (schemes S2 and S3).

Unsubstituted NDIs are colorless nonfluorescent electron traps. However, recent findings show that a minor change on the structural level suffices to introduce all of the characteristics needed for photosynthetic activity. Core substitution with electron-donating alkylamines produces compact blue, red-emitting pushpull fluorophores (excitation wavelength $\lambda_{\mathrm{ex}}=$ $638 \mathrm{~nm}$; emission wavelength $\lambda_{\text {em }}=678 \mathrm{~nm}$ ), lowers the reduction potential by $300 \mathrm{mV}$, and moves the oxidation potential within reach (22). The resulting potentials for NDI reduction to the radical anion $\mathrm{NDI}^{--}$[redox potential $\left.\left(E_{1 / 2}\right)=-710 \mathrm{mV}\right]$ and oxidation to $\mathrm{NDI}^{\cdot+}\left(E_{1 / 2}=+1290 \mathrm{mV}\right)$ appeared compatible with photoinduced charge separation in $\pi$-stack architecture 1 for electron transfer to
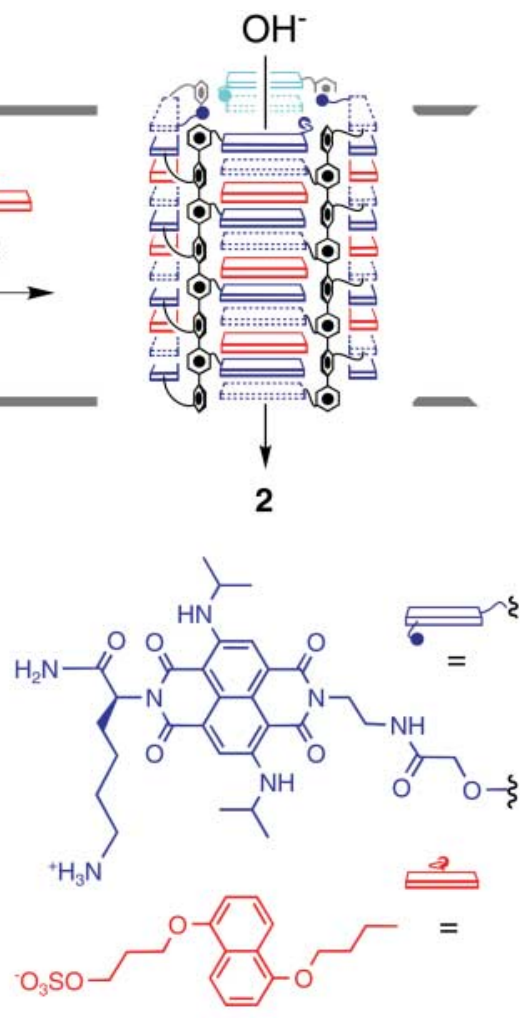

Fig. 1. Smart photosystem 1 with an indication of the ligand-gated opening into ion channel 2 and fluorometric detection of photosynthetic activity in bilayer membranes with HPTS as internal increase in $\mathrm{pH}$ after photoinduced charge separation $\left(\mathbf{1}^{\mathrm{cs}}\right)$, followed by external EDTA oxidation and internal reduction of quinones (Q). $h v$, photonic energy. 
2-sulfonato-1,4-naphthoquinone (Q) $\left(E_{1 / 2}=-60\right.$ $\mathrm{mV})$ and hole transfer to EDTA $\left(E_{1 / 2} \approx+430\right.$ $\mathrm{mV}$ ) (table $\mathrm{S} 1$ ). This photoredox process is thermodynamically unfavorable and thus converts photonic energy into chemical energy.

The photosynthetic activity of quadruple $\pi$ - $M$-helices 1 was measured in egg yolk phosphatidylcholine large unilamellar vesicles (EYPC LUVs). The vesicles were loaded with quinone acceptor $\mathrm{Q}$ and $\mathrm{pH}$ detector 8-hydroxypyrene1,3,6-trisulfonate (HPTS) and surrounded with the electron donor EDTA (Fig. 1) (4, 20). Internal quinone reduction in response to the irradiation of the externally added NDI rods 4 at $635 \mathrm{~nm}$ was detectable as an increase in intravesicular pH ("a" in Fig. 3A and fig. S4). The initial velocity of change in HPTS emission, which reflects internal proton consumption, was analyzed with Hill's equation. The value of $n=$ $3.9 \pm 1.2$ that was found indicated that the active photosystem $\mathbf{1}$ is a tetrameric supramolecule with a median effective concentration of $1.3 \mu \mathrm{M}$ (Fig. 3B) (24). Judged from exciton coupling between twisted proximal NDI chromophores throughout circular dichroism (CD) spectra (21), this tetramer is a heat-insensitive (melting temperature $\geq 70^{\circ} \mathrm{C}$ ) $M$-helix with sensitivity toward chemical denaturation [with guanidinium chloride, free energy of selforganization $\left(\Delta G^{\mathrm{H} 2 \mathrm{O}}\right) \leq 1.17 \mathrm{kcal} / \mathrm{mol}$, fig. S7]. Support for cofacial $\pi$-stacking ( 8 ) in quadruple $\pi$-M-helix 1 was secured with the appearance of a hypsochromic maximum at $598 \mathrm{~nm}$ that was absent in the absorption spec-

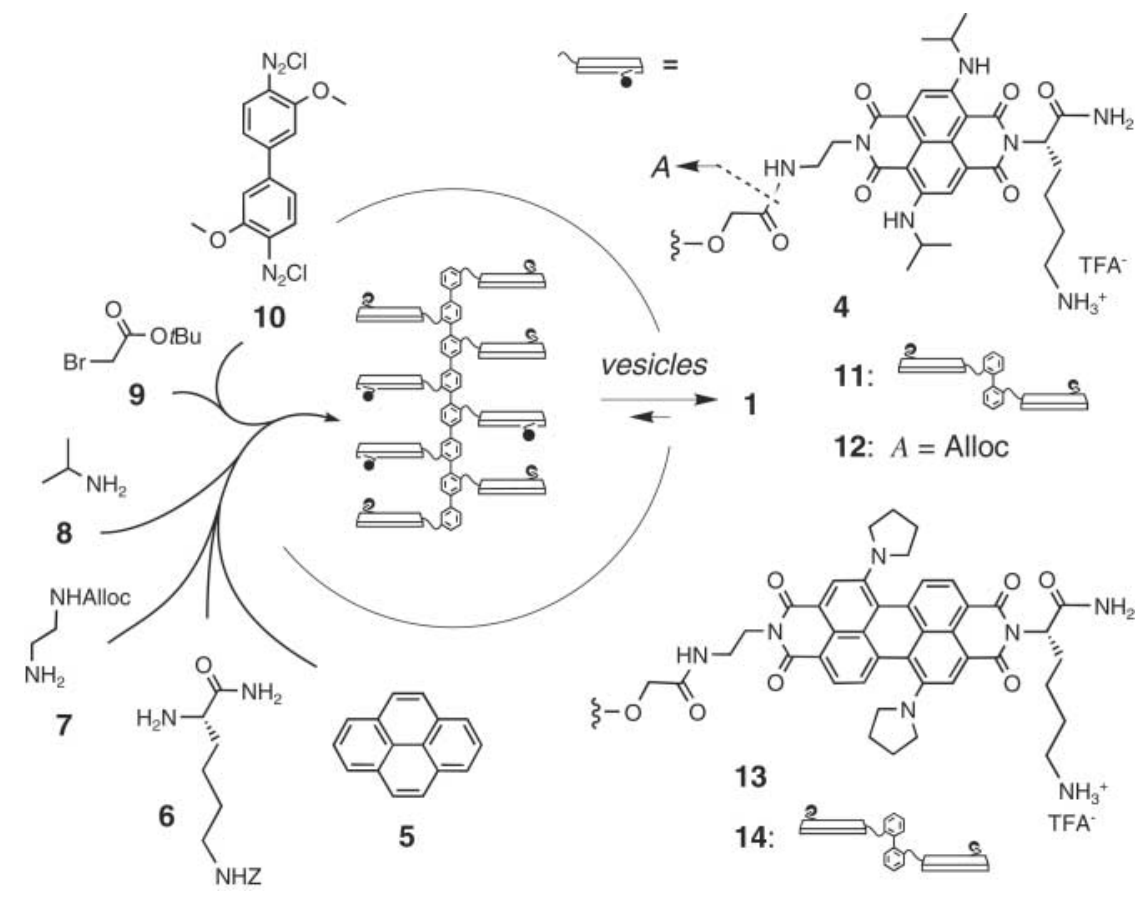

Fig. 2. Synthesis of rod 4 for self-assembly into photosystem 1 in lipid bilayers (Fig. 1) and structure of control molecules $\mathbf{1 1}$ to $\mathbf{1 4}$.

Fig. 3. Photoactivity of NDI octamer 4 (a, solid circles) with in situ opening into ion channel $\mathbf{2}$ by addition of ligand $\mathbf{3}$ (b, open squares), and comparison to NDI dimer $\mathbf{1 1}$ (c, open circles) and preformed ion channel 2 $(\mathbf{4}+\mathbf{3}, d, \times)$. (A) Change in HPTS excitation ratio $I_{462} / I_{405}\left(\lambda_{\text {em }}=\right.$ $510 \mathrm{~nm})$ as a function of irradiation time $t\left(\lambda_{\mathrm{ex}}=635 \mathrm{~nm}\right)$ in the presence of $\mathbf{1 1}(10 \mu \mathrm{M})$ or $4(1.5 \mu \mathrm{M})$ in EYPC LUVs $(\sim 65$ $\mu M$ ) with external EDTA (5 mM; $0.95 \mathrm{mM} \mathrm{K}_{\mathrm{n}} \mathrm{H}_{3-\mathrm{n}} \mathrm{PO}_{4}, 95 \mathrm{mM} \mathrm{KCl}$,
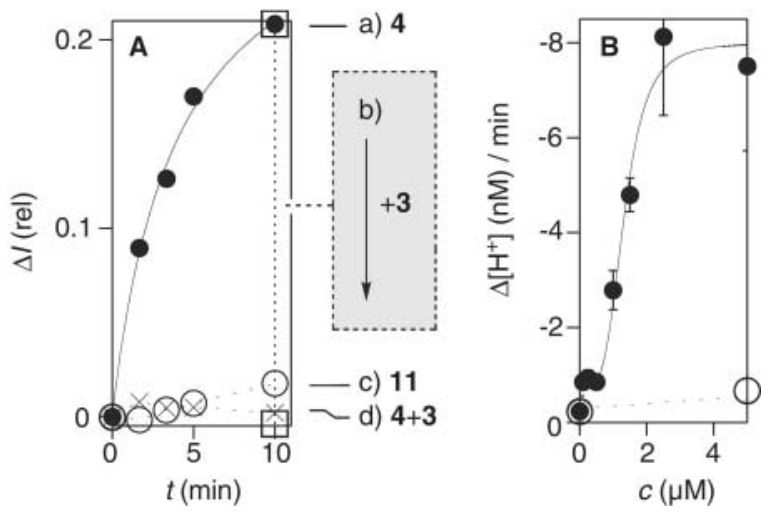

pH 7.0) and internal quinone (10 mM) and HPTS (1 mM; $100 \mathrm{mM} \mathrm{KCl}, \mathrm{pH} 7.0)$; ligand $3(20 \mu \mathrm{M}$, or gramicidin A) was added at $t=10 \mathrm{~min}$ or $t=0 \mathrm{~min}$. (B) Initial velocity of proton consumption as a function of the concentration c of $\mathbf{4}$ (solid circles) and $\mathbf{1 1}$ (open circles). Error bars indicate SE. trum of monomer $\mathbf{1 2}$ and was much weaker with dimer 11 (fig. S9).

Time-resolved fluorescence and transient absorption measurements provided direct experimental evidence that, upon irradiation of 4 , a charge-separated state characterized by a relatively long lifetime (61 ps with 4 versus 23 ps with 11) is populated almost quantitatively (>97\%, compared to $68 \%$ with 11) on an ultrafast time scale (with a distribution of time constants between $<2$ and $\sim 7$ ps) (Fig. 4 and figs. S12 to S17). These features account for the marked activity of photosystem $\mathbf{1}$. The appearance of new bands in the transient absorption spectrum of the NDI monomer 12 (trace $a$ in Fig. 4) in the presence of the electron donor $\mathrm{N}, \mathrm{N}$-dimethylaniline (DMA) confirmed that the radical anion $\mathrm{NDI}^{--}$was being detected (trace $\mathrm{b}$ in Fig. 4). The presence of the $\mathrm{NDI}^{--}$bands in the transient absorption spectra of dimer 11, as well as bands of the more pronounced, more stable octamer 4, supported photoinduced population of charge-separated state $\mathbf{1}^{\mathrm{CS}}$ (trace $\mathrm{c}$ in Fig. 4).

The addition of intercalator 3 caused a ligand-gated helix-barrel transition from photosystem 1 to ion channel $\mathbf{2}$ (fig. S5) $(20,21)$ and thus resulted in the deletion of lightinduced proton gradients [Figs. 1 and 3A (parts $\mathrm{a}$ and $\mathrm{b}$ )]; addition of the ion channel gramicidin A caused the same result. Photosynthetic activity was naturally inhibited by intercalator 3 (part d in Fig. 3A). The helix-barrel transition by intercalator 3 was also reflected in the expected $C D$ silencing $(21,25)$, together with a small increase in emission intensity that may be interpreted as hindered charge separation in barrel-stave complex $\mathbf{2}$ (figs. S6 and S8).

Control molecules 11, 13, and $\mathbf{1 4}$ were made to elucidate the dependence of photosynthetic activity of photosystem $\mathbf{1}$ on rod contraction from $p$-octiphenyl to biphenyl and on chromophore expansion from NDI to PDI. Inactivity of NDI dimer 11 (part c in Fig. 3A) coincided with $\mathrm{CD}$ silencing, the partial disappearance of the 598-nm absorption indicative of cofacial $\pi$ stacking, and incomplete photoinduced charge separation with a reduced lifetime (Fig. 4 and figs. S9 to S17). Notably, in contrast, the independence of both chiroptical and poor photosynthetic activity on rod length was found for the PDI series represented by octamer 13 and dimer 14 (fig. S20 and table S4). In agreement with molecular models (Fig. 5 and figs. S18 and S19), these results confirmed that the smart rigid-rod $\pi$-stack NDI nanoarchitecture of photosystem $\mathbf{1}$ is distinctive (that is, incompatible with the PDI series) and essential for function.

\section{References and Notes}

1. ]. Deisenhofer, H. Michel, Science 245, 1463 (1989).

2. P. Jordan et al., Nature 411, 909 (2001).

3. N. Nelson, A. Ben-Shem, Nat. Rev. Mol. Cell Biol. 5, 971 (2004). 
Fig. 4. Intensity-normalized transient absorption spectra of NDI octamer 4 (trace c) and NDI monomer 12 in the presence (trace b) or absence (trace a) of DMA in methanol after excitation with a 50-fs laser pulse at $600 \mathrm{~nm} . \Delta$ absorbance, change in absorbance; a.u., arbitrary units. The bands at 510 and above $670 \mathrm{~nm}$ seen with 4 and 12 in the presence of DMA are attributed to the $\mathrm{NDI}^{\circ-}$ radical, whereas with 12 in the absence of DMA, the 450- to 580$\mathrm{nm}$ band originates from singlet excited-state absorption. The negative signal between 600 and $670 \mathrm{~nm}$ is due to ground-state bleaching and stimulated emission. (Inset) Time profiles of the absorption due to the radical anion of octamer $\mathbf{4}$ (circles) and dimer $\mathbf{1 1}(\times)$. The lines represent best fits to the data.

Fig. 5. Molecular dynamics simulations of photosystem $\mathbf{1}$ (A) and ion channel 2 (B) with 12 ligands 3 (red) in axial view (top) and side view (bottom). In (A) (closed pore), helicity $(M)=-50.2^{\circ}$ and height $(h)=32 \AA$; in (B), inner diameter $=4.8 \AA, M=-8.8^{\circ}$, and $h \approx 40 \AA$.
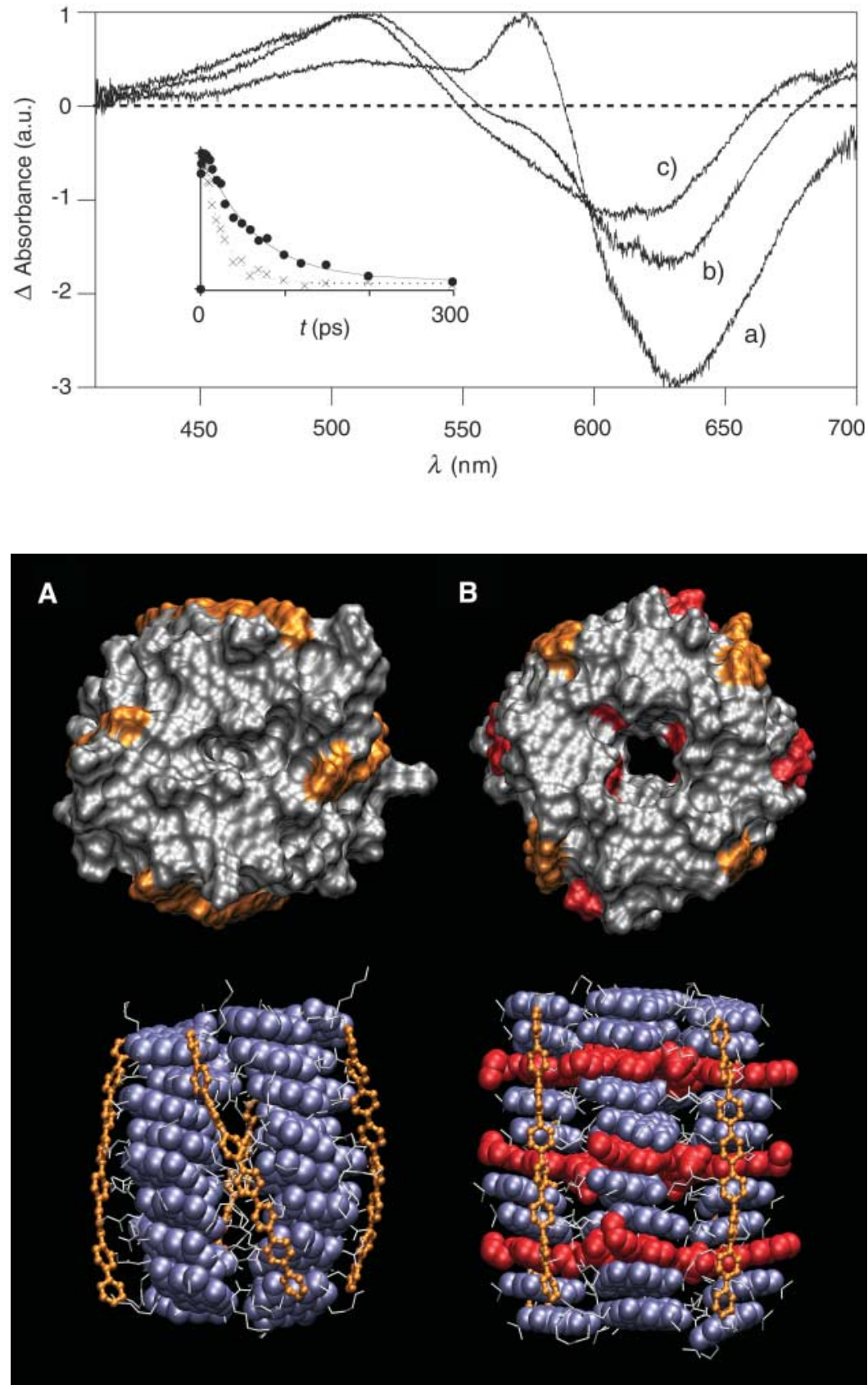

4. G. Steinberg-Yfrach et al., Nature 385, 239 (1997).

5. J. J. Grimaldi, S. Boileau, J.-M. Lehn, Nature 265, 229 (1977).

6. J. N. Robinson, D. J. Cole-Hamilton, Chem. Soc. Rev. 20 , 49 (1991).

7. L. Zhu, R. F. Khairutdinov, ]. L. Cape, J. K. Hurst, J. Am. Chem. Soc. 128, 825 (2006).

8. J. M. Giaimo, A. V. Gusev, M. R. Wasielewski, J. Am. Chem. Soc. 124, 8530 (2002).

9. M. J. Ahrens et al., J. Am. Chem. Soc. 126, 8284 (2004).

10. L. L. Miller, K. R. Mann, Acc. Chem. Res. 29, 417 (1996).

11. H. E. Katz et al., Nature 404, 478 (2000).

12. J. M. Warman et al., Chem. Mater. 16, 4600 (2004).

13. F. J. M. Hoeben, P. Jonkheijm, E. W. Meijer, A. P. H. J. Schenning, Chem. Rev, 105, 1491 (2005).

14. V. Balzani, M. Venturi, A. Credi, Molecular Devices and Machines (Wiley, Weinheim, Germany, 2003), chap. 6.

15. L. Schmidt-Mende et al., Science 293, 1119 (2001).
16. R. S. Lokey, B. L. Iverson, Nature 375, 303 (1995)

17. F. Würthner, Ed., Supramolecular Dye Chemistry (Topics in Current Chemistry Series 258, Springer, Berlin, 2005).

18. C. Wan, T. Fiebig, O. Schiemann, J. K. Barton, A. H. Zewail, Proc. Natl. Acad. Sci. U.S.A. 97,14052 (2000).

19. S. Matile, A. Som, N. Sordé, Tetrahedron 60, 6405 (2004)

20. P. Talukdar, G. Bollot, J. Mareda, N. Sakai, S. Matile, J. Am. Chem. Soc. 127, 6528 (2005)

21. P. Talukdar, G. Bollot, J. Mareda, N. Sakai, S. Matile, Chem. Eur. J. 11, 6525 (2005).

22. F. Würthner, S. Ahmed, C. Thalacker, T. Debaerdemaeker, Chem. Eur. J. 8, 4742 (2002)

23. See supporting material on Science Online.

24. To confirm the validity of this result on the structura level, a CD method for the determination of meaningful Hill coefficients for exergonic self-assembly under denaturating conditions was introduced (26)

25. J. M. Kerckhoffs et al., J. Am. Chem. Soc. 127, 12697 (2005).
26. S. Bhosale, S. Matile, Chirality, in press.

27. We thank D. Jeannerat, A. Pinto, and S. Grass for nuclear magnetic resonance measurements; P. Perrottet and the group of F. Gülaçar for mass spectrometry; and the Swiss National Science Foundation for financial support (to S.M. and E.V.). C.R. thanks the Degussa Foundation for a Ph.D. scholarship, and S.B. is a fellow of the Roche Research Foundation.

Supporting Online Material

www.sciencemag.org/cgi/content/full/313/5783/84/DC1 Materials and Methods

SOM Text

Figs. $\mathrm{S} 1$ to $\mathrm{S} 20$

Schemes $\mathrm{S} 1$ to $\mathrm{S3}$

Tables S1 to S4

References

22 February 2006; accepted 16 May 2006 $10.1126 /$ science. 1126524 